\title{
REVISÕES SISTEMÁTICAS DA LITERATURA: PARTE II
}

\section{SISTEMATIC LITERATURE REVIEWS: PART II}

\section{Regina Coeli Ruschel ${ }^{1}$ \\ Universidade Estadual de Campinas, Faculdade de Engenharia Civil, Arquitetura e Urbanismo, \\ Campinas - SP Editor Chefe parc@fec.unicamp.br \\ Letícia de Oliveira Neves ${ }^{2}$ Universidade Estadual de Campinas, Faculdade de Engenharia Civil, Arquitetura \\ e Urbanismo, \\ Campinas - SP \\ Editor Chefe \\ leticia@,fec.unicamp.br \\ Sidney Piochi Bernardini ${ }^{3}$ Universidade Estadual de Campinas, Faculdade de Engenharia Civil, Arquitetura e Urbanismo, \\ Campinas - SP Editor Chefe sidpiochi@fec.unicamp.br \\ Daniel de Carvalho Moreira 4 Universidade Estadual de Campinas, Faculdade de Engenharia Civil, Arquitetura e Urbanismo, \\ Campinas - SP Editor Chefe danmore@,fec.unicamp.br}

\section{Editorial}

O presente número completa o Volume 8 de 2017 da PARC Pesquisa em Arquitetura e Construção com a segunda parte dos artigos submetidos e aprovados na chamada específica para artigos de Revisão Sistemática da Literatura (RSL). Neste número da PARC, as RSLs são realizadas por pesquisadores dos estados brasileiros do Espirito Santo, Goiás, São Paulo e Paraná. As instituições de origem dos autores abrangem a Universidade Federal do Espírito Santo, Universidade Federal de Goiás, Universidade Estadual de Campinas, Universidade de São Paulo e a Universidade Federal do Paraná. As áreas de formação envolvidas concentram-se em Arquitetura e Urbanismo interagindo com a Engenharia Civil e a Engenharia Ambiental. O delineamento e apresentação das RSLs desenvolvidas foram baseados em Moher et al. (2015), Gough, Thomas e Oliver (2012), Dresch, Lacerda e Antunes Jr. (2015), Glass (1976) e Wohlin (2014)

A primeira RSL, da segunda parte desta edição temática da PARC, é dos autores Mariana de Cillo Malufe Spignardi e Leonardo Marques Monteiro, da Universidade de São Paulo, que correlacionam variáveis microclimáticas com a decisão pelo uso da bicicleta em centros urbanos. As variáveis microclimáticas estudadas são a temperatura média do ar, níveis de precipitação e velocidade do vento. O protocolo de revisão compreende artigos publicados nas bases Scopus, Web of Science e Infohab e nos anais dos eventos PLURIS, ENCAC e ELACAC. Os estudos analisados foram realizados majoritariamente em regiões de clima temperado úmido com verão temperado ou quente, ou em clima temperado frio, sem estação seca e com verão temperado, onde a temperatura média no mês mais quente não passa dos $22^{\circ} \mathrm{C}$. Os estudos revisados apontam que a variação na temperatura média do ar e a presença de precipitação são os aspectos de maior influência na decisão pelo uso da bicicleta, tanto para percursos utilitários quanto para recreativos. Outros aspectos que influenciam o uso da bicicleta, como os socioeconômicos e culturais, também são abordados de forma a complementar. Verifica-se a necessidade de avaliações que considerem a influência conjunta das variáveis microclimáticas, estabelecendo uma relação com a sensação e preferência térmicas dos ciclistas, assim como estudos em regiões com outros climas para uma avaliação mais ampla sobre a decisão pelo uso da bicicleta em centros urbanos.

\section{How to cite this article:}

RUSCHEL, Regina C. et al. Revisões sistemáticas da Literatura: Parte II. PARC Pesquisa em Arquitetura e Construção, Campinas, SP, v. 8, n. 4, p. 217-219, dez. 2017. ISSN 1980-6809. Disponível em: <https://periodicos.sbu.unicamp.br/ojs/index.php/parc/article/view/8652036>. Acesso em: 29 jan. 2018. doi:http://dx.doi.org/10.20396/parc.v8i4.8652036. 
A RSL dos autores Paulo José Lima Perillo, Marcus André Siqueira Campos e Loyde Vieira de AbreuHarbich, da Universidade Federal de Goiás, sistematiza resultados de estudos sobre o conforto térmico de salas de aula. $\mathrm{O}$ protocolo de revisão compreende artigos publicados nas bases de dados Science Direct, Scopus e Engineer Village. Foi possível identificar os métodos utilizados pelos pesquisadores, as estratégias de climatização dos ambientes analisados, o valor amostral e os resultados encontrados. Observou-se que os estudos que utilizam o modelo adaptativo (ATC - Adaptive Thermal Comfort) têm maior compatibilidade com as sensações térmicas dos usuários que os estudos que utilizam o modelo racional (RTC - Rational Thermal Comfort). Os autores apontam para a necessidade da realização de pesquisas em regiões de clima tropical considerando a adaptação do usuário ao clima e a aplicação de mais de um método de avaliação de conforto para verificar a adequação da problemática ao contexto brasileiro e a necessária divulgação em revistas internacionais indexadas.

A RSL dos autores Jéssica Cristine da Silva Fonseca Matos e Paulo Sergio Scarazzato, da Universidade Estadual de Campinas, buscou esclarecer o quanto e como está sendo incluída a questão da iluminação natural nos estudos sobre processo de projeto em arquitetura. $\mathrm{O}$ protocolo de revisão envolveu buscas de artigos publicados entre 2012 e 2017 nas bases de dados Scopus, Science Citation Index Expanded, Science Direct Journals $e$ Civil Engineering Abstracts. Para o desenvolvimento desta revisão foram analisados apenas os artigos com ênfase em metodologias e ferramentas para análise e projeto de iluminação. Os resultados dos estudos analisados foram sistematizados considerando as fases inicias de projeto, simulações computacionais utilizadas, metodologias para a tomada de decisão de projeto e a influência do usuário no processo. Os autores sugerem adiar para etapas posteriores de projeto a utilização de softwares de simulação e aplicar na fase inicial do projeto a sensibilidade e expertise do projetista considerando aspectos da luz do dia e das necessidades humanas. São também sugeridas características: de metodologias de projeto, de interatividade no processo, de ferramentas e de automação de sistemas de sombreamento.

Bruna Vogt Bär e Sergio Fernando Tavares, da Universidade Federal do Paraná, por meio da RSL delineiam o estado da arte do comportamento hidrológico de telhados verdes no Brasil. O protocolo de busca abrangeu as bases de dados Banco de Teses e Dissertações da CAPES e SciELO e a máquina de busca na internet do Google Acadêmico. Observou-se uma diversidade de abordagens e diferenças entre os componentes dos telhados verdes utilizados. Trabalhos referentes à capacidade de retenção de águas pluviais apresentam conclusões concordantes. Entretanto, os resultados dos estudos referentes à qualidade das águas escoadas por essas coberturas são variantes ou contraditórios. Em geral, concorda-se que a camada de substrato e a de vegetação tendem a liberar maiores concentrações de substâncias, como fósforo, nitrogênio, ferro e nitrito; e podem ainda diminuir a qualidade da água escoada. Observa-se que métodos de pesquisa padronizados auxiliariam na compatibilização de resultados, e poderiam contribuir para a construção de uma evidência cientifica eficaz de adaptação e desempenho dessas coberturas para regiões brasileiras.

A RSL de Marcella Ruschi Mendes Saade e Vanessa Gomes da Silva, da Universidade Estadual de Campinas, com Maristela Gomes da Silva, da Universidade Federal do Espírito Santo, visa delinear um panorama científico do uso de métodos de distribuição em Avaliações do Ciclo de Vida (ACV). Para tal, avaliou-se a frequência de escolha dos métodos nos estudos publicados no período de 2006 a 2016 na base de dados da Springer, que abriga o periódico International Journal of Life Cycle Assessment. Os resultados revelaram uma falta de consenso entre praticantes de ACV. As autoras avaliam que o problema de distribuição de impactos na ACV é tipicamente solucionado de forma contrária ao encaminhamento teórico proposto em norma, sugerindo uma oportunidade de reflexão e reformulação.

A RSL de Katrin Rappl e Leandro Silva Medrano, da Universidade de São Paulo, busca mapear métodos e pesquisas existentes sobre análise e avaliação de projetos habitacionais de interesse social que possam ser aplicados na Pré-Construção. O protocolo de busca abrangeu estudos científicos publicados nas bases de dados SciELO, Scopus, Web of Science, Science Direct e Biblioteca Digital Brasileira de Teses e Dissertações, assim como aqueles obtidos pela máquina de busca do Google Scholar. Os autores observam que não existe um consenso sobre como avaliar projetos habitacionais, visto que uma avaliação pode tratar de diferentes dimensões, escalas de aproximação e etapas do projeto, bem como 
considerar aspectos tangíveis e/ou intangíveis; os quais implicam em formas distintas de aferir. Do mesmo modo, também não há consenso entre os estudos na forma pela qual são estabelecidos os parâmetros de análise ou avaliação. Como contribuição, os autores sugerem, a partir dos resultados e juntamente com a fundamentação, formas de aferir um projeto arquitetônico, de estabelecer parâmetros de análise ou avaliação e apontam às bases conceituais envolvidas. $\mathrm{O}$ estudo assim identifica potencial para o desenvolvimento de pesquisas e do conhecimento metodológico no campo denominado de
Avaliação Pré-Construção (APC), sobretudo quando considerada a demanda nacional por habitações de qualidade destinadas à população de menor renda.

Estes dois números temáticos da PARC, sobre RSLs, buscam fomentar na área de Arquitetura e Construção uma prática já estabelecida nas áreas de Saúde e da Ciência da Computação. Assim, apresenta-se uma base metodológica e exemplos de RSLs que a mapeiam, avaliam criticamente, consolidam e agregam resultados de estudos primários identificando ênfases, tendências e lacunas de pesquisa.

\section{Agradecimentos}

Agradecemos aos avaliadores pelo tempo despendido e valiosas considerações tecidas sobre os artigos submetidos.

\section{Referências}

DRESCH, Aline; LACERDA, Daniel Pacheco; ANTUNES JR, José Antônio Valle. Design Science Research. 1. ed. Cham: Springer International Publishing, 2015. 176 p. Disponível em: <http://www.springer.com/gp/book/9783319073736>. .978-3-31907373-6.

GLASS, G.V. Primary, secondary, and meta-analysis of research. Educational Researcher, v.5, n. 10, p. 3-8, 1976. http://www.jstor.org/stable/1174772.

GOUGH, David; THOMAS, James; OLIVER, Sandy. Clarifying differences between review designs and methods. Systematic Reviews, v. 1, n. 1, p. 1 , 2012. doi: http://dx.doi.org/10.1186/2046-4053-1-28.

MOHER, D. et al. Preferred reporting items for systematic reviews and meta-analyses: the PRISMA statement. Tradução de Taís Freire Galvão e Thais de Souza Andrade Pansani. Retro-traduzido David Harrad. Epidemiol. Serv. Saúde, Brasília, v. 24, n. 2, p. 355 - 342, abr/jun. 2015. http://doi.org/ 10.1371/journal.pmed.1000097

WOHLIN, C. Guidelines for snowballing in systematic literature studies and a replication in software engineering. In: INTERNATIONAL CONFERENCE ON EVALUATION AND ASSESSMENT IN SOFTWARE ENGINEERING, $18,2014$. London. Proceedings... New York: ACM, 2014. p. 1-10.

\footnotetext{
${ }^{1}$ Regina Coeli Ruschel

Engenheira Civil. Doutor em Engenharia Elétrica e da Computação (UNICAMP). Livre Docente em Projeto Auxiliado por Computador (UNICAMP). Professora e Pesquisadora Colaboradora (FEC-UNICAMP) Endereço postal: Av. Albert Einstein, 951, Campinas, SP, Brasil, CEP 13.083-852.

\section{${ }^{2}$ Letícia de Oliveira Neves}

Arquiteta Urbanista. Doutora em Arquitetura, Tecnologia e Cidade pela Universidade Estadual de Campinas. Professora Doutora na Faculdade de Engenharia Civil, Arquitetura e Urbanismo da UNICAMP. Endereço postal: Av. Albert Einstein, 951, Campinas, SP, Brasil, CEP 13.083-852.
}

\section{${ }^{3}$ Sidney Piochi Bernardini}

Arquiteto Urbanista. Doutor em Arquitetura e Urbanismo (FAU-USP). Professor Doutor na Faculdade de Engenharia Civil, Arquitetura e Urbanismo da UNICAMP. Endereço postal: Av. Albert Einstein, 951, Campinas, SP, Brasil, CEP 13.083-852.

\footnotetext{
${ }^{4}$ Daniel de Carvalho Moreira

Arquiteto Urbanista. Doutor em Engenharia Civil (UNICAMP). Professor Doutor II na Faculdade de Engenharia Civil, Arquitetura e Urbanismo da UNICAMP. Endereço postal: Av. Albert Einstein, 951, Campinas, SP, Brasil, CEP 13.083-852.
} 